\title{
Análise numérica de um perfil aerodinâmico utilizando a Plataforma OpenFOAM
}

\author{
Numerical analysis of an aerodynamic profile using the Open FOAM \\ Plataform
}

\author{
João de Sousa Bomfim \\ Neto \\ Instituto Federal do Ceará \\ - IFCE joaodesousa1@ \\ gmail.com \\ Luana Sousa Silva \\ Universidade de Fortaleza \\ - Unifor \\ luusousa17@gmail.com

\section{Francisco de Assis Leandro Filho \\ Instituto Federal do Ceará - IFCE leandro.filho@ifce. edu.br} \\ Daniel Barros de Freitas \\ Universidade de Fortaleza \\ - Unifor \\ danielbfreitas@unifor.br \\ Brígida Miola Rocha \\ Universidade de Fortaleza \\ - Unifor \\ bmiola@unifor.br
}

\begin{abstract}
Resumo
A mecânica dos fluidos é uma das áreas mais abrangentes nas engenharias, sendo, quase sempre, um dos primeiros contatos do graduando com uma disciplina específica. Sua aplicação vai desde a multiplicação de força em um braço hidráulico pelo princípio de Pascal à construção de grandes barragens. O presente trabalho está inserido na subárea da mecânica dos fluidos que estuda o comportamento de corpos imersos em escoamento não estacionário, a dinâmica dos fluidos domputacional ou CFD (computational fluid dynamics). Neste caso, foi analisado um perfil aerodinâmico utilizando a plataforma livre OpenFOAM para as simulações e visualização do comportamento das camadas de fluido. O perfil aerodinâmico e seu respectivo domínio foram criados em software livre de CAD gerador de malhas GMSH, para posterior importação para o OpenFOAM, em que foram extraídas as informações acerca das forças aerodinâmicas no perfil, apresentadas através de gráficos com o auxílio do software GNUPLOT.
\end{abstract}

Palavras-chave: Aerodinâmica. CFD. OpenFOAM.

\begin{abstract}
Fluid mechanics is one of the most comprehensive areas in engineering, being, often, one of the first contacts of the graduate with a specific discipline. Its application range from the multiplication of force in a hydraulic arm by the principle of Pascal to the construction of great dams. This work is a part of the fluid mechanics area, that studies the behavior of bodies immersed in non-stationary flow, computational fluid dynamics, or CFD. An aerodynamic profile was analyzed using the OpenFOAM free platform for the simulations and preview of the fluid layers behavior. The aerodynamic profile and its respective domain were created in the free CAD software mesh generator GMSH, for subsequent import to OpenFOAM, where the information about aerodynamic forces in the profile was extracted, exposed through graphs with the aid of GNUPLOT software.
\end{abstract}

Keywords: Aerodynamics. CFD. OpenFOAM

\section{Introdução}

A mecânica dos fluidos é a ciência que estuda o comportamento dos fluidos, bem como as leis que regem esse comportamento. A gama de aplicações da mecânica dos fluidos na engenharia é bastante ampla, como no estudo de máquinas hidráulicas, esforços em barragens, ventilação e aerodinâmica. Dentro desta vasta ciência, o presente estudo se concentra em escoamentos externos, que são escoamentos sobre corpos imersos em um fluido sem fronteira. O escoamento sobre uma esfera, ou sobre um corpo carenado, é exemplo de escoamento externo (FOX, 2009).

O estudo de escoamentos externos é de alta importância para a engenharia aeronáutica, de forma a analisar o escoamento do ar em vários componentes de uma aeronave. Boa parte do conhecimento que se tem hoje acerca do assunto foi desenvolvido a partir da resolução de problemas de aerodinâmica. Contudo, existem diversas áreas de interesse na aplicação de conhecimentos relativos ao escoamento externo, como: o escoamento em torno de uma pá de turbina, escoamento sobre a superfície de um automóvel, escoamento de transporte de glóbulos vermelhos, escoamento sobre edificações, dentre outros (POTTER; WIGGERT, 2004). 
A análise de efeitos aerodinâmicos em mecânica dos fluidos é algo complexo e requer muito conhecimento específico. As leis físicas que governam os fenômenos associados à aerodinâmica recaem numa matemática um tanto complexa, com várias equações diferenciais parciais geradas a partir de simplificações das equações de Navier-Stokes, tornando a análise analítica impraticável, como pode se verificar em bibliografias básicas, como a de Franco Brunetti (2008). Outro modo de análise é o experimental, de forma que, em um túnel de vento adaptado à situação, consegue-se levantar os dados necessários à análise, podendo ser incrementado ainda o uso de sensores. Este modo, porém, pode se tornar demasiado caro, pois seriam necessários grandes túneis de vento para grandes perfis, o que ainda necessitaria de grandes instalações, tornando o processo dispendioso.

Para se realizar uma análise em que se entre num meio termo entre os dois exemplos citados acima, recorre-se aos métodos numéricos computacionais, em que é necessário certo grau de dedicação mental e dispor de um aparato computacional razoável e compatível com o problema em questão, pois o tempo gasto é consideravelmente menor do que na resolução de equações e muito menos dispendioso do que o método experimental, semelhante ao trabalho de Passos (1984). Cabe destacar que esse método só existe e é possível utilizá-lo porque é conhecida a física-matemática do problema e há a possibilidade de experimentação posterior, afim de comprovar o resultado numérico.

Diante do exposto, este trabalho tem como objetivo analisar um perfil aerodinâmico, usando apenas um computador com o sistema operacional Linux Ubuntu ${ }^{\circledR}$ e conhecimentos relativos aos fatores físicos envolvidos com a dinâmica do escoamento com foco no perfil imerso. O desenho do perfil e a geração da malha foram feitos no software GMSH, cuja principal funcionalidade é esta. Em seguida, a malha foi importada para o OpenFOAM, responsável pela simulação propriamente dita, que com o auxilio dos softwares ParaView (incluso no pacote do OpenFOAM, 2011) e GNUPLOT, permitiram a visualização dos resultados na forma gráfica, reforçando a numérica cedida pelo OpenFOAM.

A dinâmica de fluidos computacional (CFD) é utilizada para resolver numericamente problemas de escoamento de fluidos, sendo presente em trabalhos que buscam estudar uma vasta gama de aplicações da física e da engenharia. É um ramo da mecânica de fluidos que utiliza análises numéricas e estruturas de dados para resolver e analisar problemas que envolvem fluxos de fluidos (CHUNG, 2010). Os computadores são usados para realizar os cálculos necessários para simular a interação de líquidos e gases com superfícies definidas por condições de contorno. A base fundamental de quase todos os problemas CFD são as equações de Navier-Stokes, que definem muitos fluxos de fluídos monofásicos (gases ou líquidos, mas não ambos). Essas equações podem ser simplificadas pela remoção de termos que descrevem ações viscosas para produzir as equações de Euler (CHUNG, 2010). Uma base dos conceitos físicos envolvidos no processo serão desenvolvidos a seguir.

Comparando-se a um sólido, pode-se ressaltar a definição de fluido a partir de sua forma. Um sólido tem uma forma própria, enquanto um fluido tem forma indefinida, dada de acordo com o recipiente que o contém (BRUNETTI, 2008). Essa é uma definição generalista, que orienta sobre a identificação do que é um fluido.

Contudo, em relação à um solido, deve haver uma distinção mais específica, associada à forças e tensões aplicadas, a saber: como os fluidos atingem,ou não, o equilíbrio após aplicadas essas forças e tensões. Essa distinção é obtida a partir da clássica "experiência das duas placas" (Figura 1), em que um sólido é colocado entre as duas placas e, posteriormente, um fluido (a), sendo ambos sujeitos à uma força tangencial (b) na placa superior estando a inferior engastada.

Figura 1 - Experimento das duas placas planas

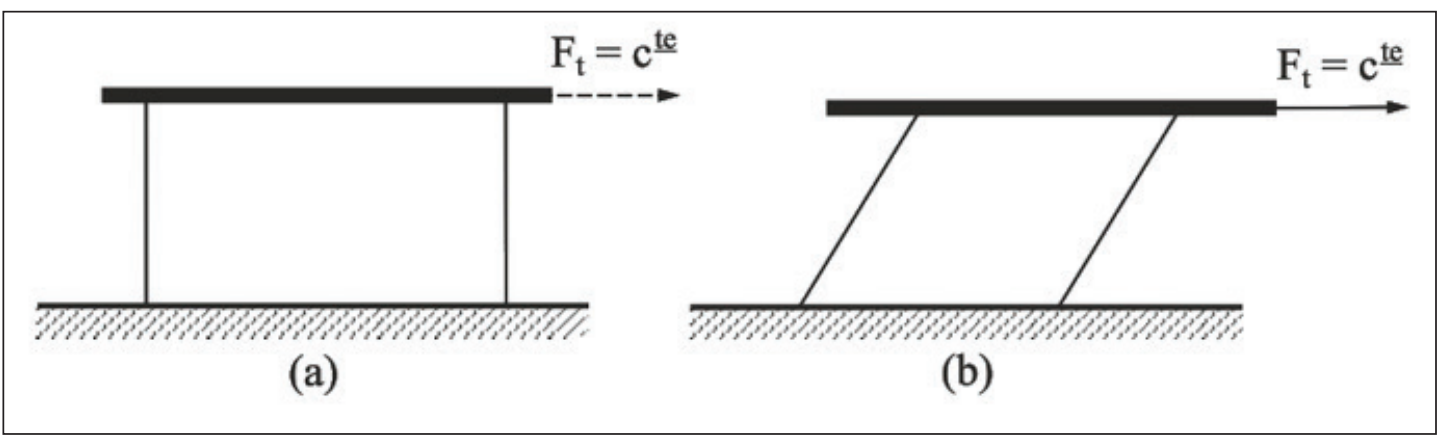


Observa-se que, ao aplicar uma força pequena em um sólido, este sofre uma pequena deformação angular e atinge uma nova posição de equilíbrio estático, diferente de um fluido, que se deforma continuamente, não se encontrando, posteriormente, em uma nova configuração de equilíbrio estático enquanto a força constante é aplicada.

Pode ser considerado fluido ideal aquele que não resiste ao escoamento, ou seja, tem viscosidade nula. Com isso, o fluido não perde energia por atrito. Obviamente, não existe este tipo de fluido. No entanto, para análises menos minuciosas, ou em situações hipotéticas, convém adotar o fluido de trabalho como ideal.

Para este trabalho, o fluido é considerado incompressível, caso em que tem volume constante com a variação de pressão, consequentemente do tempo. Matematicamente:

$$
\frac{\partial \rho}{\partial t}=0
$$

Na realidade, esta condição inexiste. Fluidos líquidos, porém, têm comportamento muito próximo ao caso em questão, assim como os gases também, desde que não sofram grandes pressões. Logo, para fluidos e gases em baixas pressões, a incompressibilidade é uma condição aceitável.

A dinâmica dos fluidos estuda a interação entre um corpo e um meio fluido no qual o corpo está imerso, existindo entre eles um movimento relativo, em que é adotado como referencial o corpo (sólido). Sendo assim, não importa se apenas o corpo ou fluido, ou ambos, estão em movimento, pois, para o observador, o sólido estará sempre em repouso e o fluido escoará contra o corpo.

Graças à impenetrabilidade do sólido, é visível que a massa de fluido em escoamento seja perturbada nas adjacências do corpo. O movimento de contorno da massa escoante na superfície do sólido provocará o aparecimento de uma força. Caso seja analisado o sólido de perfil de modo a possibilitar a decomposição em duas componentes, estas serão (Figura 2):

- Resistência ao avanço, ou força de arrasto $\left(F_{a}\right)$, paralelas às linhas de corrente do escoamento;

- Força de sustentação $\left(F_{S}\right)$, que é a componente normal ou perpendicular às linhas de corrente.

Figura 2 - Forças de arrasto e sustentação atuando sobre um corpo carenado.

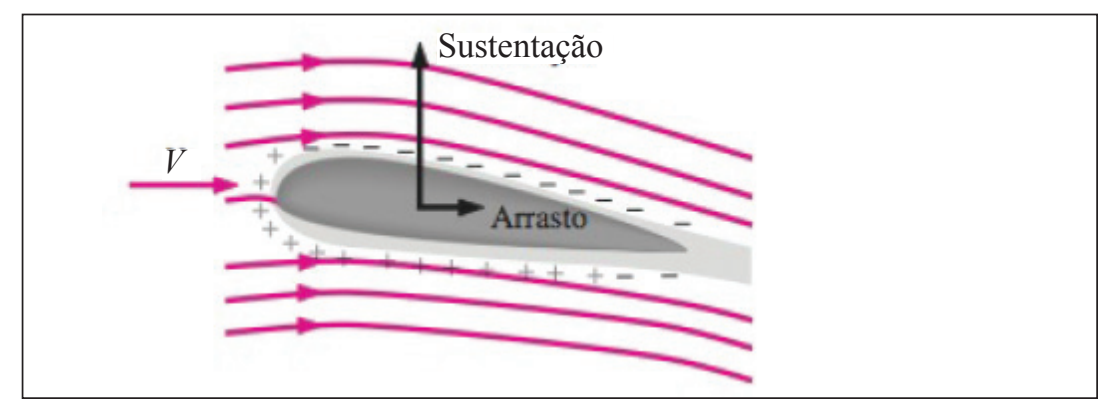

Porém, para o desenvolvimento de estimativas dessas forças (geralmente determinadas de maneira empírica), algumas hipóteses são assumidas, de forma a facilitar o trabalho:

- Fora do campo de perturbação, as linhas de corrente são paralelas;

- Fora do campo de perturbação, o diagrama de velocidades é uniforme;

- O escoamento é não-transiente;

- O fluido é incompressível;

- As diferenças de cotas não influenciam na determinação das diferenças de pressão.

No estudo de escoamento de fluidos, é conveniente correlacionar o máximo possível de variáveis em uma só, de forma a simplificar os cálculos e economizar tempo e pesquisa. Esse é o objetivo da adimensionalização de parâmetros. A correlação de variáveis pode ser escrita como razão entre os diversos tipos de forças que um fluido sofre durante o escoamento (POTTER; WIGGERT, 2004). São algumas delas: 
Força de Pressão:

$$
F_{P}=\Delta p A \approx \Delta p l^{2}
$$

Força Viscosa:

$$
F_{\mu}=\tau A=\mu \frac{d v}{d y} A \approx \mu \frac{v}{l} l^{2}=\mu l v
$$

Força Inercial:

$$
F_{I}=m v \frac{d v}{d s} \approx \rho l^{3} v \frac{v}{l}=\rho l^{2} v^{2}
$$

Ainda existindo as forças centrífuga, de compressibilidade, etc.

Onde:

$l=$ Comprimento do elemento

$m=$ Massa

$s=$ Superfície

$v=$ Velocidade do escoamento

Observou-se que a laminação, ou turbulência do escoamento, era função da força de inércia e da força viscosa. Quão maior fosse a força - a força inercial em relação à força resistente ao escoamento, ou seja, a força viscosa -, mais o escoamento tenderia ao regime turbulento. Assim, deu-se origem ao número de Reynolds (Re), como a seguinte razão entre as duas forças acima mencionadas:

$$
\operatorname{Re}=\frac{F_{I}}{F_{\mu}}=\frac{\rho l^{2} v^{2}}{\mu l v}=\frac{\rho l v}{\mu}=\frac{l v}{v}
$$

\section{Materiais e métodos}

O OpenFOAM (originalmente FOAM) foi criado por Henry Weller no final de 1980, com o objetivo de realizar simulações mais flexíveis e capazes que as realizadas pelos softwares da época, que utilizavam FORTRAN como linguagem. A plataforma é predominantemente CAE (computer aided engineering), ou seja, é destinada a fazer simulações e testes relativos à problemas de engenharia, e utiliza linguagem de programação $\mathrm{C}++$. Sendo assim, o software necessita de um modelo em CAD (computer aided design) já pronto com sua devida malha (mesh) processada em elementos finitos (2D), ou volumes finitos (3D). Assim, esta é convertida e importada para o software para a realização da análise numérica (JASAK et al, 2007).

Para essa simulação, foi criada uma malha de um aerofólio, inicialmente, usando o pacote GMSH. As malhas foram criadas através do perfil NACA 5012 (Figura 3). 
Figura 3 - Aerofólio "malhado" com elementos finitos (visualização isométrica).

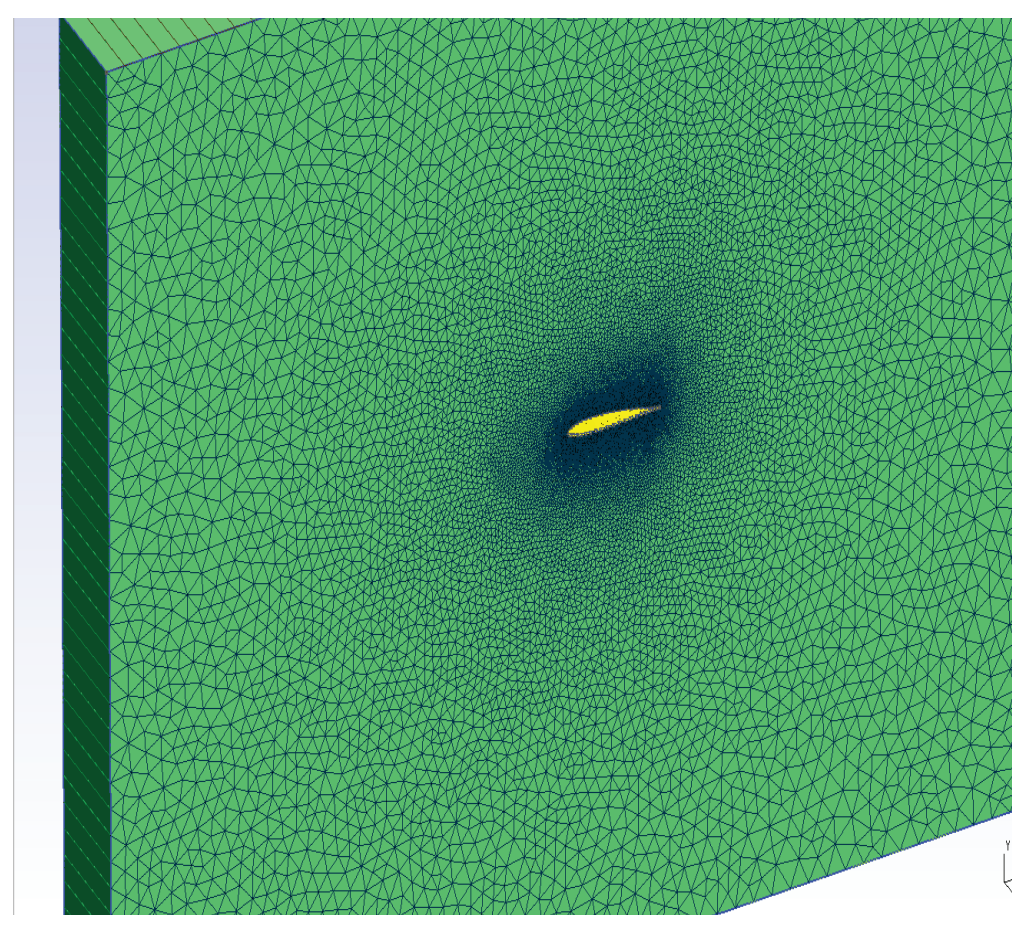

A malha tem mais pontos perto da superfície e menos perto dos limites. Isto é controlado pelo comprimento característico dos vários pontos que foram definidos, especificando-se 0,005 para as superfícies de aerofólio e 0,2 para as bordas.

Após a exportação da malha, foi feita a simulação do escoamento externo de um fluido através da plataforma OpenFOAM. Feita a modelagem computacional do problema físico e as simulações, alguns dados de velocidade, campo de força e pressão foram extraídos e plotados em gráficos usando o software GNUPLOT. O tempo empregado na simulação foi de $500 \mathrm{~s}$, com o passo de tempo de $1 \mathrm{~s}$ (como consequência, cada iteração será para o intervalo de 1s), e o perfil foi inclinado em relação ao eixo x (horizontal) em oito graus através da decomposição do vetor velocidade, cujo valor modular foi de $4 \mathrm{~m} / \mathrm{s}$. Escolheu-se uma pequena inclinação a fim de se observar as sucintas mudanças no escoamento mediante a incrementação de cada passo de tempo.

\section{Resultados e discussão}

Para se visualizar o perfil, algumas imagens foram geradas via print screen, sendo essas imagens da décima leitura do programa, no tempo final (500 s) e estando o perfil com uma inclinação de oito graus em relação ao vento relativo (o ar é o fluido de escoamento, neste caso). 
Para o contorno de pressão:

Figura 4 - Campo de pressão ao redor do perfil, com escala e em vista bidimensional.

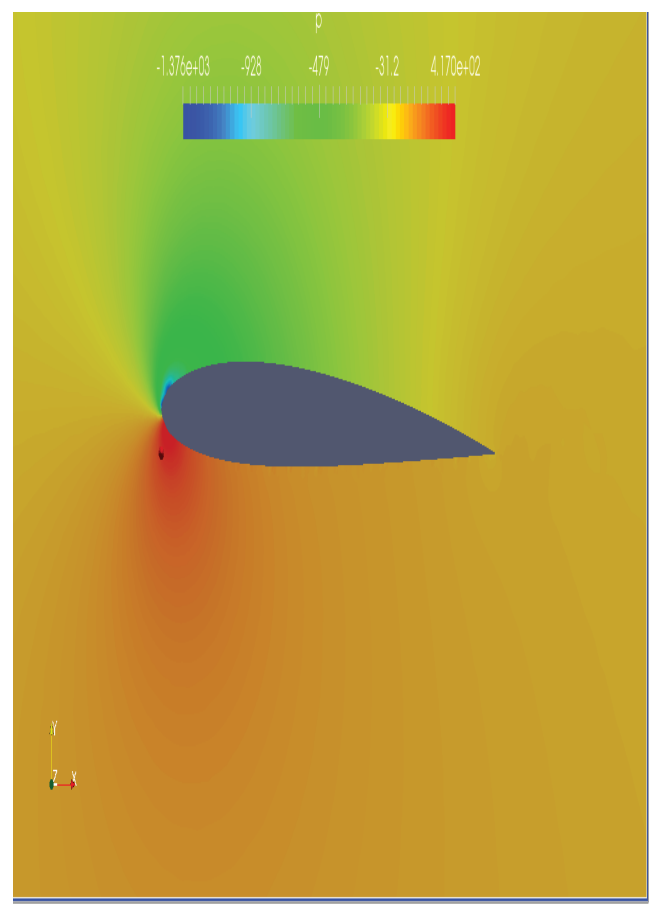

Como mostra a figura 4, as pressões mais acentuadas localizam-se próximas ao bordo de ataque na parte inferior por causa da inclinação positiva para cima de oito graus. Mesmo com essa pequena inclinação, percebe-se um gradiente acentuado de pressão entre a parte inferior e superior do bordo de ataque.

Para a velocidade resultante:

Figura 5 - Campo de velocidades ao redor do perfil, com escala e em vista bidimensional.

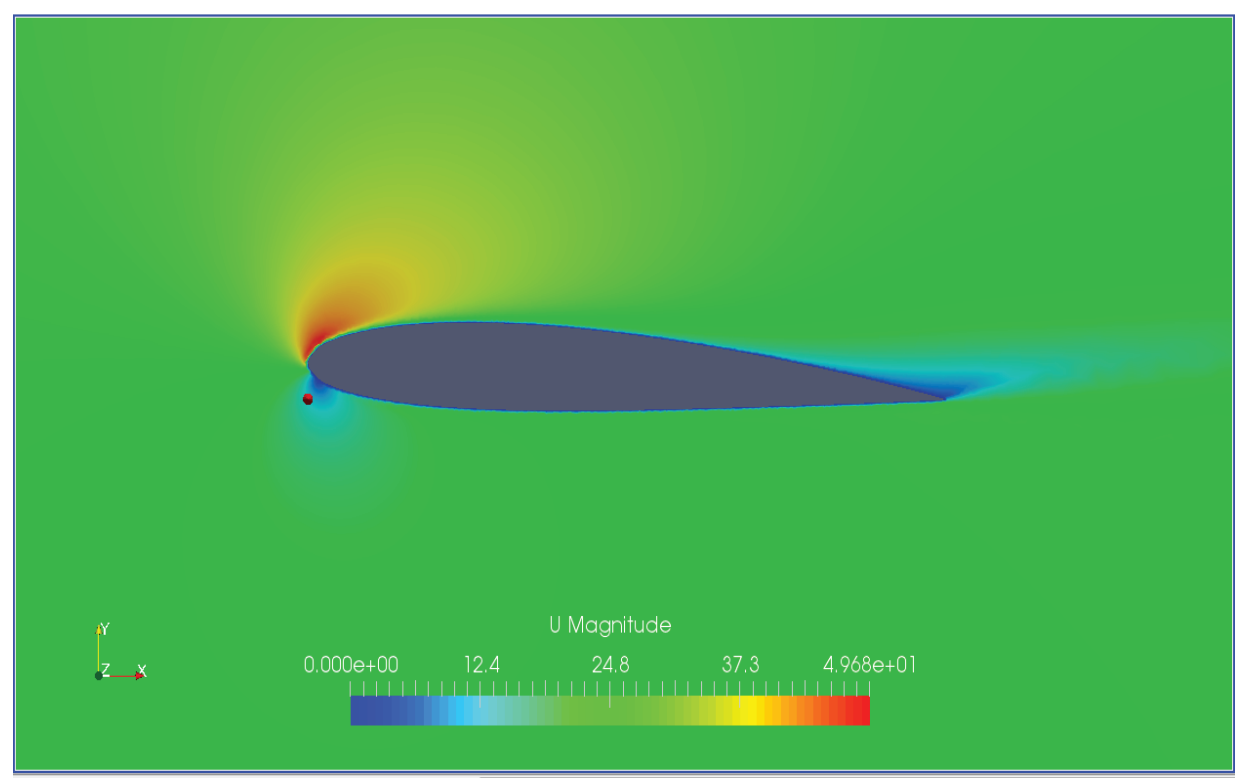


Na figura 5 observa-se o campo de velocidade se contrapondo ao gradiente de pressão da figura, o que retrata bem a compensação entre pressão e velocidade durante o escoamento de um fluido sobre um corpo aerodinâmico: em regiões onde há maior pressão, há menor velocidade, e vice-versa.

Para a visualização dos contornos de pressão, podemos verificar a simulação na figura 6:

Figura 6 - Contornos de pressão do escoamento.

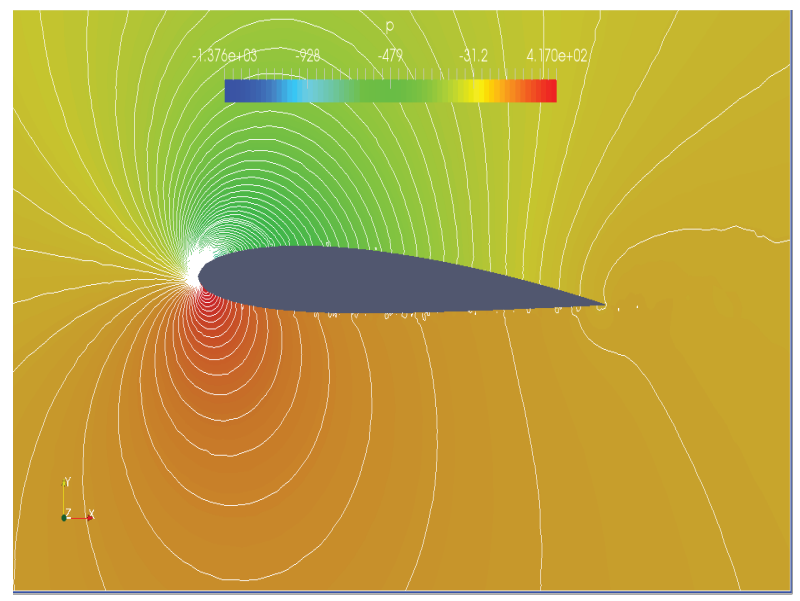

A figura 6 ressalta os contornos de pressão através de linhas que delimitam regiões com valores semelhantes. Ainda é possível ter uma visualização 3D da superfície do perfil e ver a distribuição de pressão sobre ele. No extradorso:

Figura 7 - Pressão no extradorso do aerofólio, com escalas e em 3D.

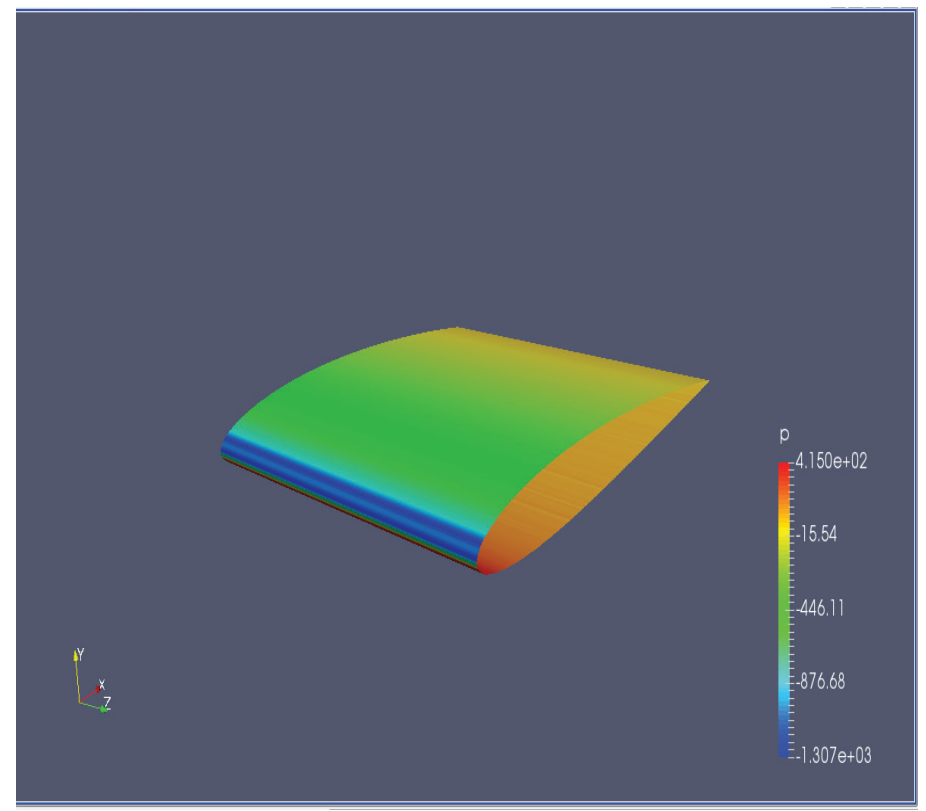

Na figura 7, uma representação tridimensional do gradiente de pressão na parte superior do perfil pode ser vista. Neste caso, a representação bidimensional já seria o bastante, mas, em situações em que o perfil não apresenta alta simetria e o escoamento não é uniforme, essa representação se faz necessária. 
No intradorso:

Figura 8 - Pressão no intradorso do aerofólio, com escalas e em 3D.

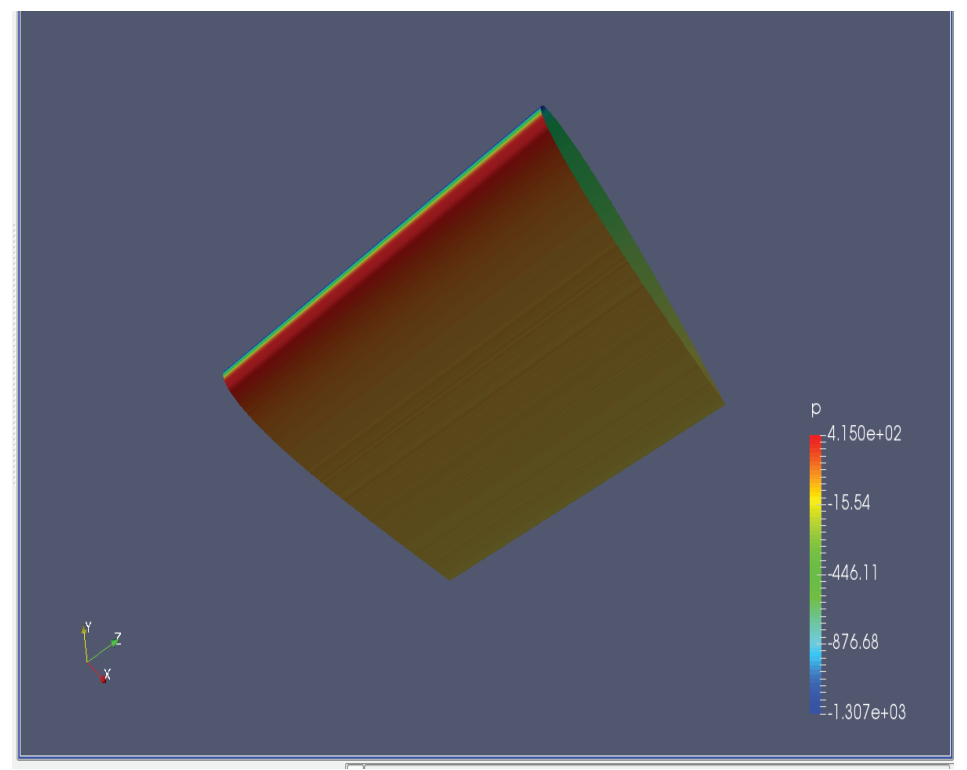

Na figura 8 é possível ver o efeito contrário que acontece no extradorso. No intradorso, as pressões são maiores e velocidades, menores, principalmente perto do bordo de ataque do perfil (como já foi mencionado anteriormente).

Por último, dentro do ParaView, pode-se também plotar um gráfico em tempo real das grandezas físicas que se queira. Na figura 9, o gráfico é relativo a uma seção imediatamente perto do bordo de ataque, indicada pela linha vertical branca:

Figura 9 - Gráficos de velocidade e pressão em tempo real (simultâneos à simulação)

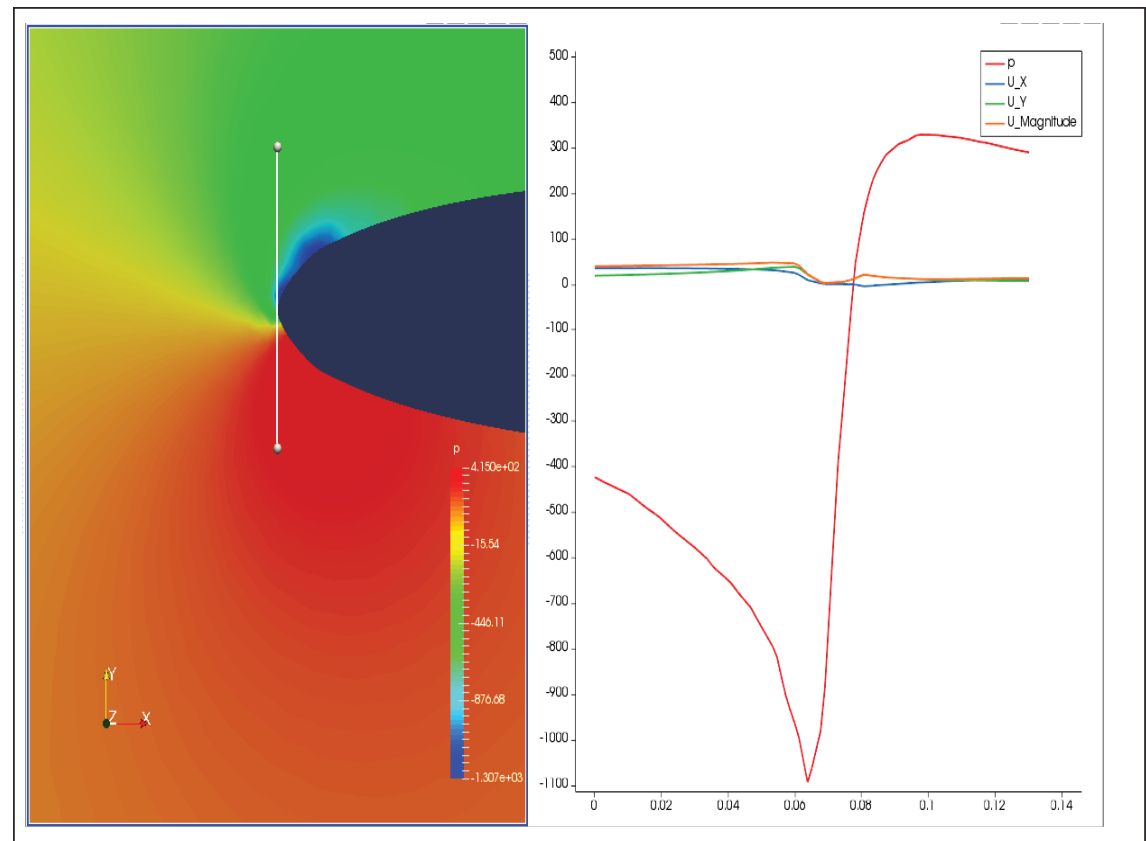

Os dados de convergência de pressão e velocidade foram plotados com relação aos pontos mencionados na seção, sendo mostrada a alteração do controlDict. Os dados de força de arrasto e sustentação foram plotados com os dados obtidos devido à alteração no controlDict. Os gráficos que seguem foram plotados com o programa GNUPLOT, através de scripts desenvolvidos para adequar os dados extraídos em .dat ao programa. 
As figuras 10 a 12 retratam a saída dos dados de força e campos de pressão e velocidade, bem como sua convergência. Forças:

Figura 10 - Desenvolvimento das forças em relação ao tempo de simulação.

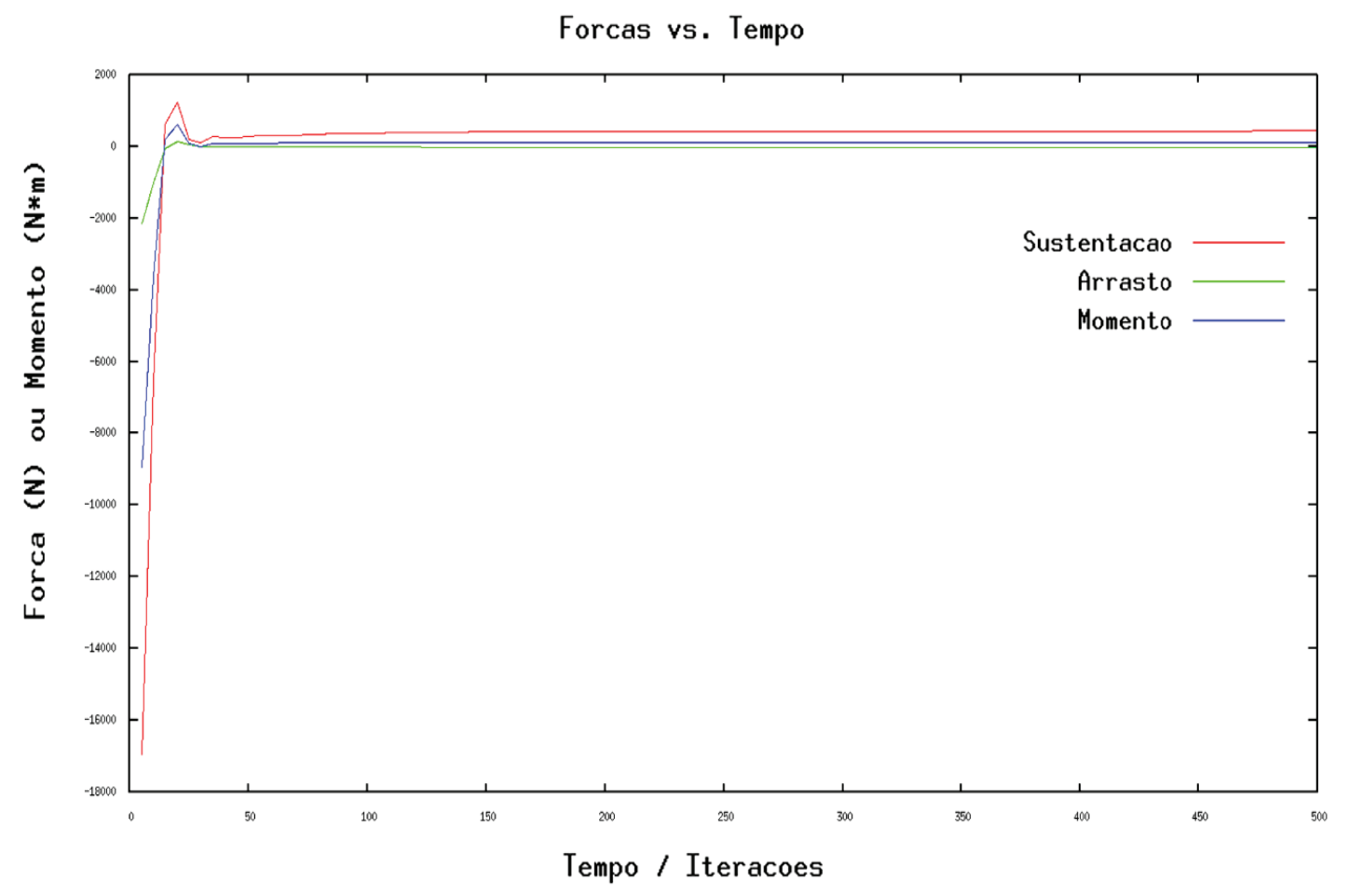

A figura 10 mostra a curva de força e seu desenvolvimento em relação à cada iteração, ou passo de tempo (sabendo que cada passo de tempo vale $1 \mathrm{~s}$, então cada iteração vale $1 \mathrm{~s}$ ). O comportamento da curva de sustentação condiz com a física, pois, no momento inicial do escoamento, o choque de fluido com o perfil (inclinado levemente para cima) faz com que tenha uma força gerada para cima (sustentação positiva), atingindo um pico e sendo estabilizada ao passo que o escoamento também se estabiliza.

O arrasto mostra um desenvolvimento inicial e mantém uma constância logo em seguida, não apresentando um pico, como a sustentação, devido à baixa inclinação do perfil (oito graus) e ao fato deeste ser carenado (baixo efeito do arrasto). $\mathrm{O}$ momento indicado refere-se à força resultante agindo sobre um braço de alavanca ao longo do corpo, algo não interessante para este trabalho. 
João de Sousa Bomfim Neto, Luana Sousa Silva, Francisco de Assis Leandro Filho, Daniel Barros de Freitas, Brígida Miola Rocha

A seguir, é mostrada a convergência de pressão e velocidade nos pontos sonda (probes), respectivamente:

Figura 11 - Convergência de pressão pr i+amn ñ̃

\section{CONVERGENCIA}

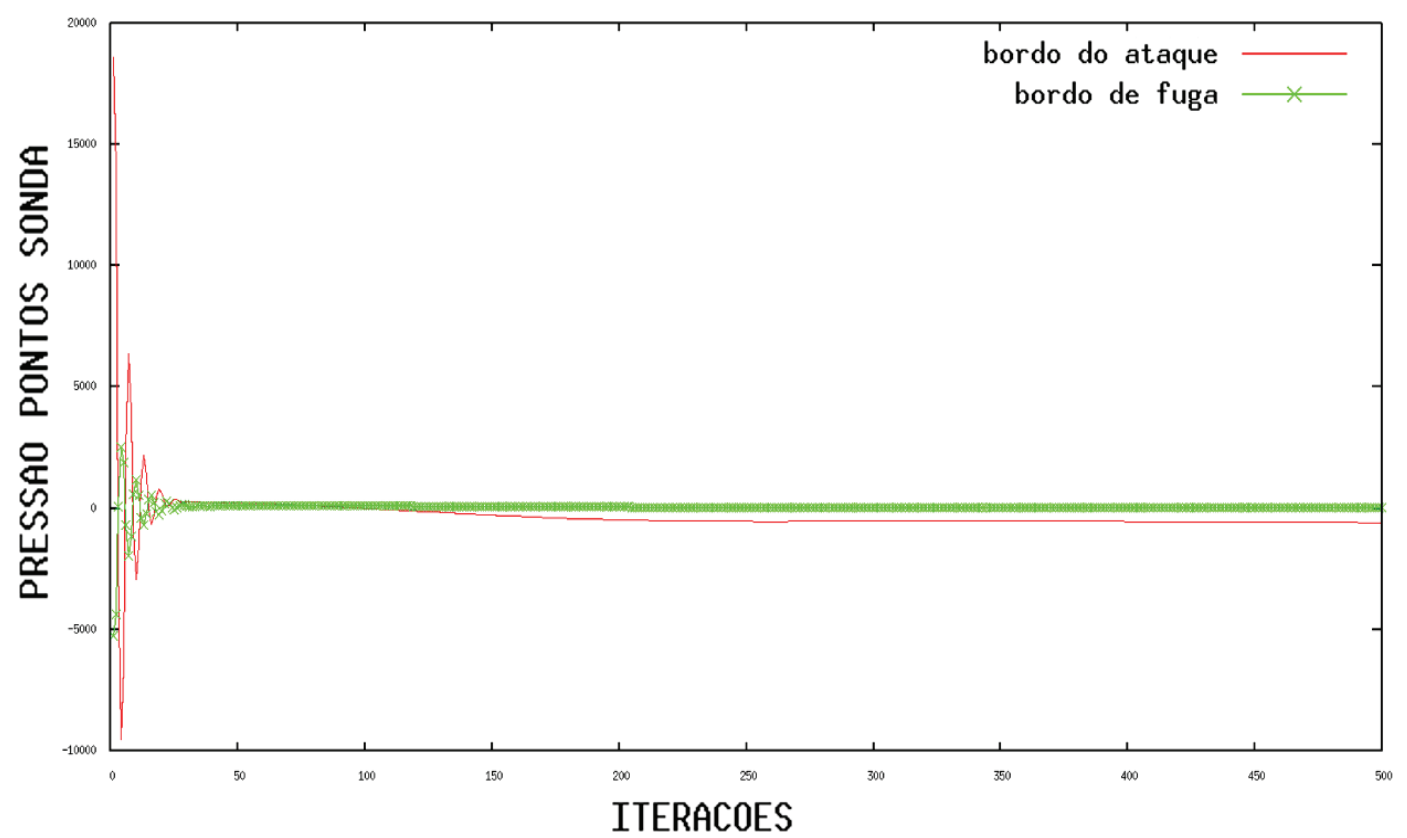

Figura 12 - Convergência de velocidade

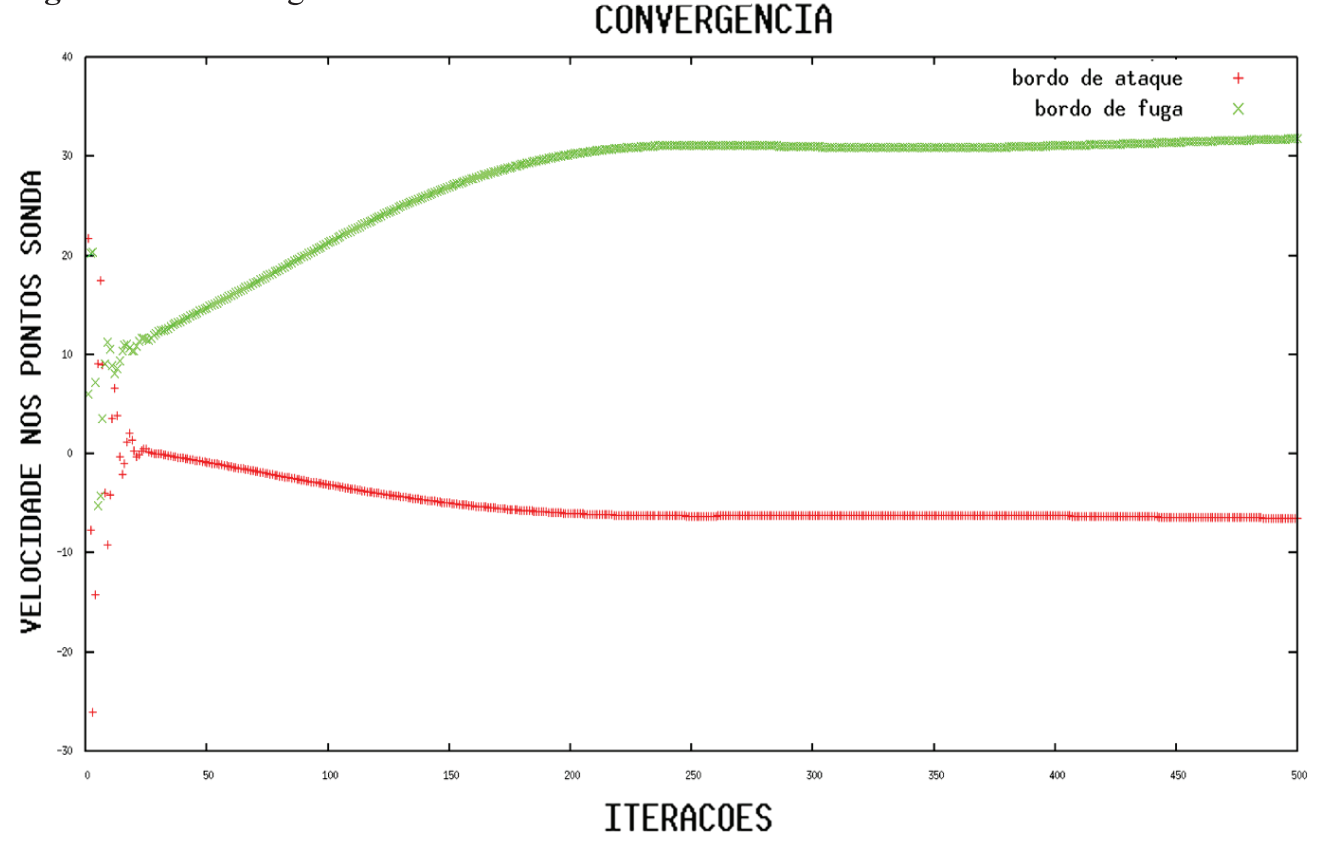

As figuras 11 e 12 retratam a convergência dos pontos, ilustrando bem a turbulência inicial antes de o regime atingir a característica laminar. Nota-se que, nas mediações dos $25 \mathrm{~s}$, o escoamento deixa de ter características turbulentas e passa para o regime laminar devido a continuidade das linhas traçadas. Na proximidade dos $300 \mathrm{~s}$, o escoamento, além de laminar, mostra-se semi-constante, o que é retratado pelas linhas cada vez mais retas. 


\section{Conclusão}

Com os resultados obtidos, pode-se concluir que os softwares de simulação ajudam muito na visualização e entendimento de fenômenos físicos, bem como no seu desenvolvimento com o passar do tempo. Para o manuseio de um software de manipulação em CFD, como neste caso, é necessário um conhecimento prévio do caso a ser tratado, ou pelo menos do básico em dinâmica dos fluidos e aerodinâmica, se considerarmos a comparação com o uso de um software comercial;

Caso o software seja livre, é muito provável que seja adaptado ao sistema operacional Linux e necessite de programação em código aberto, o que exigirá do usuário conhecimentos em linguagem computacional. Dessa forma, pode ainda ser comprovada a importância da multidisciplinaridade ao se deparar com uma ferramenta como foi usada neste trabalho, não só em questão de desenvolvimento de software, mas no próprio manuseio, ressaltando a importância da associação entre mecânica dos fluidos e métodos numéricos.

Com relação aos gráficos apresentados, nota-se que houve uma convergência de pontos de forma a construir uma curva, indicando que o modelo matemático e a forma de construção estão coerentes. A validação deste caso deveria ser feita experimentalmente para enriquecer ainda mais o trabalho, porém, seria necessário um aporte financeiro considerável no quesito computacional, estrutural e sensorial. Apesar disso, os resultados se mostraram válidos e satisfatórios, o que é possível afirmar devido ao conhecimento da física envolvida já comprovada através da literatura básica.

\section{Referências}

BRUNETTI, F. Mecânica dos fluidos. 2. ed. São Paulo: Pearson, 2008.CHUNG, T. J. Computational Fluid Dynamics. Cambridge University Press, 2010.

FOX, R. W.; PRITCHARD, P. J.; MCDONALD, A. T. Introduction to fluid mechanics. 7. ed. Hoboken: John Wiley \& Sons, 2009.

JASAK, H. et al. OpenFOAM: A C++ library for complex physics simulations. In: INTERNATIONAL WORKSHOP ON COUPLED METHODS IN NUMERICAL DYNAMICS, 2007, Croatia. Proceedings... Croacia: IUC Dubrovnik , 2007. p. 1-20. Disponível em: <https://www.researchgate.net/publication/228879492_OpenFOAM_A_C_library_for_ complex_physics_simulations $>$. Acesso em: 24 nov. 2017.

OpenFOAM Foundation. OpenFOAM User Guide. 2011. Disponível em: <https://openfoam.org/version/2-0-1/>. Acesso em: 27 nov. 2017.

PASSOS, J. C. Rotor eólico darrieus: um estudo esperimental. 1984. 117 f. Dissertação (Mestrado em Engenharia Mecânica) - Universidade Federal do Rio de Janeiro, Rio de Janeiro, 1984. - Dúvida

POTTER, M. C.; WIGGERT, D. C. Mecânica dos Fluidos. São Paulo: Thomson Pioneira, 2004. 
Sobre os autores

\section{João de Sousa Bomfim Neto}

Possui graduação em Engenharia Mecânica pela Universidade de Fortaleza (2016), com pesquisas desenvolvidas em transferência de calor, método dos elementos finitos, mecânica analítica, mecânica dos fluidos, simulações computacionais com ênfase na utilização de softwares livres e de programação aberta e ensino na engenharia. Atualmente é aluno do Programa de Pós-graduação em Energias Renováveis - IFCE do Instituto Federal de Educação, Ciência e Tecnologia do Ceará, IFCE.

\section{Luana Sousa Silva}

Possui graduação em Engenharia Ambiental pela Universidade de Fortaleza (2017). Tem experiência na área de Engenharia Sanitária, com ênfase em Engenharia Sanitária, atuando principalmente nos seguintes temas: Análises físicoquímicas de solo e água, mecânica dos fluidos, e desenvolvimento sustentável.

\section{Francisco de Assis Leandro Filho}

Graduado em Física pela Universidade Estadual do Ceará (2010) e Mestre em Engenharia Mecânica pela Universidade Federal do Ceará (2012). Atualmente é aluno do Programa de Pós-Graduação em Física da Universidade Federal do Ceará em nível de Doutorado e Professor de Ensino Básico, Técnico e Tecnológico, EBTT do Instituto Federal de Educação, Ciência e Tecnologia do Ceará, IFCE - Campus Jaguaruana.

\section{Daniel Barros de Freitas}

Mestre em Engenharia de Teleinformática na Universidade Federal do Ceará (2012), na Área de Concentração Eletromagnetismo Aplicado, Linha de Pesquisa - Sistemas e Dispositivos de Micro-ondas e Antenas. Especialista em Ensino e Prática de Física na Universidade Federal do Ceará (2007). Licenciado em Física pela Universidade Federal do Ceará (2001).

\section{Brígida Miola Rocha}

Doutora em Ciências Marinhas pela Universidade Federal do Ceará e Senckenberg Forschungsinstitut - Alemanha. Mestre em Ciências Marinhas (LABOMAR - UFC) e Bacharel em Química com habilitação em Química Industrial (UFC). Vinculada ao Laboratório de Geologia e Geomorfologia Costeira e Oceânica (LGCO-UECE). Professora integral do Centro de Ciências Tecnológicas da Universidade de Fortaleza e Coordenadora Pedagógica do curso Tecnólogo em Energias Renováveis. 